\title{
Review Article \\ History, Introduction, and Kinetics of Ion Exchange Materials
}

\author{
Sanjeev Kumar and Sapna Jain \\ University of Petroleum and Energy Studies, Dehradun, Uttarakhand 248007, India \\ Correspondence should be addressed to Sapna Jain; sapnaj22@gmail.com
}

Received 20 May 2013; Revised 14 August 2013; Accepted 15 August 2013

Academic Editor: Roberto Comparelli

Copyright (c) 2013 S. Kumar and S. Jain. This is an open access article distributed under the Creative Commons Attribution License, which permits unrestricted use, distribution, and reproduction in any medium, provided the original work is properly cited.

\begin{abstract}
During the last few decades, ion exchange materials have evolved from laboratory tool to industrial products with significant technical and commercial impact. The current paper briefly summarizes the history of the development of the ion exchange materials. The paper defines the ion exchange materials and their types. The paper signifies the kinetics involved in the ion exchange process with description of factors affecting the rate of ion exchange. The mechanism of ion exchange has also been delineated through schematic diagram, illustrating that there are two types of diffusion, film and particle controlled diffusion. A brief of mathematical approach for kinetics of ion exchange has also been incorporated.
\end{abstract}

\section{Introduction}

1.1. Historical Background. Whenever an ion is removed out of an aqueous solution and is replaced by another ionic species, it is generally referred to as "ion exchange." It is an ancient technique, in cultural heritage. Renaissance pottery of Mediterranean basin consisting of glass thin film had heterogeneous distribution of silver and copper nanoparticles of varied sizes, deposited on ceramic substrate [1-3]. The copper/silver deposition was obtained by putting a mixture of copper and silver salts and oxides, together with vinegar, ochre, and clay, on the surface of a previously glazed pottery. Then the whole system was heated to about $600^{\circ} \mathrm{C}$ in a reducing atmosphere produced by the introduction of smoking substances in the kiln. In these conditions, an ion exchange process between metal ions and alkali ions in the glass was induced. Later on, the metal ions followed by a reduction aggregated, remaining trapped within the first layer.

Even before the sunup of the human civilization, the ion exchange technique can be traced back to the Holy bible, mentioning the preparation of drinking water by "Moses" from brackish water.

Although, the exchange of cations as a phenomenon was first discovered slightly more than hundred years ago, two English agricultural chemists, Thompson and Way, noted that certain soils had a greater ability than others to absorb ammonia from fertilizers $[4,5]$. They found that complex silicates in the soil performed an ion exchange function. They were able to prepare materials of this type in the laboratory from solutions of sodium aluminate and sodium silicate. In 1906, Rober Gans used materials of this type for softening water and for treating sugar solutions [6]. The first organic ion exchangers were synthesized in 1935; Adams and Holmes found that the crushed phonograph records exhibit ion exchange properties [7]. In the middle 1940's, ion exchange resins were developed based on the copolymerization of styrene crosslinked with divinylbenzene. These resins were very stable and had much greater exchange capacities than their predecessors. The polystyrene-divinylbenzene-based anion exchanger could remove all anions, including silicic and carbonic acids. This innovation made the complete demineralization of water possible.

The discovery of ion exchangers was predetermined by the overall progress in fundamental science in the middle (Michael Faraday, concept of ions) and late (Svante Arrhenius, theory of electrolytic solutions) eighteenth century. These ideas were crucial for the scientific discovery of ion exchangers, because such materials, both organic and inorganic, are essentially polyelectrolytes. This means that they can be considered as consisting of two ions of opposite charge. Contrary to conventional electrolytes, ions of one charge are fixed to a polymeric (organic ion exchangers) or crystalline (inorganic ion exchangers) structure. Like any ionic compound, an ion exchange material can dissociate and 
participate in ion exchange reactions. However, dissociation does not result in dissolving of the material. This causes many specific phenomena and many possibilities to design heterogeneous systems with ionic properties (Table 1).

1.2. Ion Exchange Materials. Ion exchange is a chemical reaction in which free mobile ions of a solid, the ion exchanger, are exchanged for different ions of similar charge in solution. The exchanger must have an open network structure, either organic or inorganic, which carries the ions and which allows ions to pass through it.

An ion exchanger is a water-insoluble substance which can exchange some of its ions for similarly charged ions contained in a medium with which it is in contact; this definition is all embracing. Referring to a "substance" rather than a compound includes many exchangers-some of them are natural products which do not have a well defined composition. The term "medium" acknowledges that ion exchange can take place in solution both aqueous and nonaqueous, in molten salts, or even in contact with vapours. The definition is not limited to solids, since some organic solvents which are immiscible with water can extract ions from aqueous solution by an ion exchange mechanism [10].

The definition also indicates something about the process of ion exchange. Basically it consists of contact between the exchanger and the medium in which the exchange takes place. These are usually a solid ion exchanger and an aqueous solution. The fact that ions are exchanged implies that the exchanger must be ionized, but only one of the ions in the exchanger is soluble. That ion can exchange, while the other, being insoluble, cannot do so.

If we represent the ion exchanger as $\mathrm{M}^{+} \mathrm{X}^{-}$to show that it is ionized $-\mathrm{M}^{+}$being the soluble ion-and imagine it placed in a solution of the salt NY, which ionizes to give the ions $\mathrm{N}^{+}$ and $\mathrm{Y}^{-}$, the exchange reaction can be written as follows:

$$
\mathrm{M}^{+} \mathrm{X}^{-}+\mathrm{N}^{+}+\mathrm{Y}^{-} \longrightarrow \mathrm{N}^{+} \mathrm{X}^{-}+\mathrm{M}^{+}+\mathrm{Y}^{-}
$$

This resembles a simple displacement reaction between two salts MX and NY. Since the ion $\mathrm{Y}^{-}$takes no part in the reaction it can be omitted from both sides of the equation, which then simplifies to

$$
\mathrm{M}^{+} \mathrm{X}^{-}+\mathrm{N}^{+} \longrightarrow \mathrm{N}^{+} \mathrm{X}^{-}+\mathrm{M}^{+}
$$

In this example cations are exchanged, similarly an analogous equation can be written for an ion exchange when the insoluble ion in the exchanger is the cation.

There is no strict difference between ion exchange resin and chelating resins because some polymers can act as chelating or nonchelating substance depending on the chemical environment. Ion exchange resembles sorption because both are surface phenomenon, and in both cases a solid takes up a dissolved species. The characteristic difference between these two phenomena is in stoichiometric nature of ion exchange. Every ion removed from the solution is replaced by an equivalent amount of same charge (Figure 1). In sorption on the other hand a solute is usually taken up nonstoichiometrically without being replaced.
Conventionally, ion exchange materials are classified into two categories, cation exchangers and anion exchangers, based on the kind of ionic groups attached to the material.

Cation exchange materials containing negatively charged groups like sulphate, carboxylate, phosphate, benzoate, and so forth are fixed to the backbone material and allow the passage of cations but reject anions, while anion exchange materials containing positively charged groups like amino group, alkyl substituted phosphine $\left(\mathrm{PR}_{3}{ }^{+}\right)$, alkyl substituted sulphides $\left(\mathrm{SR}_{2}{ }^{+}\right)$, and so forth are fixed to the backbone materials and allow passage of anions but reject cations. There are also amphoteric exchangers that are able to exchange both cations and anions simultaneously. However, the simultaneous exchange of cations and anions can be more efficiently performed in mixed beds that contain a mixture of anion and cation exchange resins or passing the treated solution through several different ion exchange materials. There is one other class of ion exchanger known as chelating ion exchanger. Many ions accept lone pair of electrons from ligands establishing covalent like bonds, called coordination bonds. Depending on the number of coordination bonds, the ligand is called monodentate, bidentate, or polydentate. Coordination interactions are highly specific. An example of a coordination compound is the coordination of a metal ion with ethylene diamine tetra acetic acid (EDTA) (Figure 2).

Organic structure of functional groups often contains nitrogen, oxygen, and sulphur atoms, which are electron donor elements (Figure 3).

1.3. Types of Ion Exchange Material. Based on origin the ion exchange materials can be classified as natural and synthetic (Figure 4).

1.4. Natural Organic Products. Several natural organic materials possess ion exchange properties or can be given to them by simple chemical treatment. Plant and animal cells act as ion exchangers by virtue of the presence of carboxyl groups of amphoteric proteins. These carboxyl groups, $\left(-\mathrm{CO}_{2} \mathrm{H}\right)$, and phenolic groups, $(-\mathrm{OH})$, are weakly acidic and will exchange their hydrogen ions for other cations under neutral or alkaline conditions. The humins and humic acids found in natural soil "humus" are examples of this class of exchanger; the partially decayed and oxidized plant products contain acid groupings. Several organic products are marketed which are based on treated cellulose, either in fibre form for use in ion exchange columns or as filter papers for ion exchange separations on paper. Many ion exchangers have been prepared from other natural materials such as wood, fibres, peat, and coal by oxidation with nitric acid or, better still, with concentrated sulphuric acid when the strongly acid sulphonic acid group, $\left(-\mathrm{SO}_{3} \mathrm{H}\right)$ is introduced into the material. The latter process was particularly successful with sulphonated coals. These can exchange in acid solution, because the exchanging group itself is ionized under these conditions, whereas the weaker carboxylic and phenolic groups are not. All these materials have certain disadvantages; however, they tend to colour the solutions which are treated, and their properties are difficult to reproduce because of the difficulty of controlling the treatment they are given. 
TABLE 1: The main development steps of ion exchange $[8,9]$.

\begin{tabular}{|c|c|c|}
\hline Scientist name/source & Breakthrough in field of ion exchange & Year \\
\hline Bible & Moses experiments water debitteri. & $\sim 1400 \mathrm{BC}$ \\
\hline Aristotle & $\begin{array}{l}\text { Aristotle finds that sea water loses part of its salt contents when percolated through } \\
\text { certain sand. }\end{array}$ & $\sim 330$ BC \\
\hline H. S. Thompson & $\begin{array}{l}\text { Thompson passed a solution of manure through a filter made of ordinary garden } \\
\text { soil and found that the ammonia was removed from solution. }\end{array}$ & 1845 \\
\hline $\begin{array}{l}\text { H. S. Thompson and J. } \\
\text { T. Way } \\
\text { J. Spence }\end{array}$ & $\begin{array}{l}\text { Recognition of the phenomenon of ion exchange and a description of its basic } \\
\text { characteristics. } \\
\text { The ion exchange property of soils was found to be based on their containing small } \\
\text { amounts of zeolites. }\end{array}$ & $1848-1852$ \\
\hline H. Eichorn & $\begin{array}{l}\text { Proved that the adsorption of ions by clays and zeolites constitutes a reversible } \\
\text { reaction. }\end{array}$ & 1858 \\
\hline J. Lemberg & $\begin{array}{l}\text { Zeolites recognized as carriers of base exchange in soils; equivalence of exchange of } \\
\text { bases proved. }\end{array}$ & 1876 \\
\hline $\begin{array}{l}\text { F. Harm, A. Rumpher, } \\
\text { S. Mayert, and K. } \\
\text { Halse }\end{array}$ & $\begin{array}{l}\text { Artificial zeolites used for removal of potassium from sugar juices. First synthetic } \\
\text { industrial ion exchanger. Manufacture of sulphonated coals and suggestion for the } \\
\text { removal of potassium from sugar juices. }\end{array}$ & 1901-1902 \\
\hline R. Gans & $\begin{array}{l}\text { Discovered that the zeolites could be used to soften hard water. He also invented } \\
\text { processes for synthesizing zeolites and designed the equipment-the zeolite water } \\
\text { softner used for the recovery of gold from sea water. }\end{array}$ & 1905 \\
\hline O. Folin, R. Bell & The first analytical application of ion exchange. & 1917 \\
\hline J. Whitehorn & The first use of ion exchange in column chromatography. & 1923 \\
\hline A. Bahrdt & The first use of ion exchange column for anion analysis. & 1927 \\
\hline O. Leibknecht & $\begin{array}{l}\text { The entirely new types of cation exchangers were developed. Not only could they be } \\
\text { used in the sodium cycle when regenerated with salt, but also in the hydrogen cycle } \\
\text { when regenerated with an acid. One group of these cation exchangers was the } \\
\text { carbonaceous type, which was made by the sulphonation of coal. }\end{array}$ & 1934-1939 \\
\hline $\begin{array}{l}\text { B. A. Adams and E. L. } \\
\text { Holmes }\end{array}$ & Synthesis of the first organic ion exchanger. & 1934-1935 \\
\hline G.F. D’alelio & Invention of sulphonated polystyrene polymerization cation exchangers. & 1942 \\
\hline $\begin{array}{l}\text { G. E. Boyd, J. } \\
\text { Schubert, and A. W. } \\
\text { Adamson }\end{array}$ & $\begin{array}{l}\text { Demonstration of the applicability of ion exchange for adsorption of fission } \\
\text { products in traced amounts. }\end{array}$ & 1942 \\
\hline C. H. Mcburney & Invention of aminated polystyrene polymerization anion exchangers. & 1947 \\
\hline A. Skogseid & Preparation of potassium-specific polystyrene cation-exchanger chelating resin. & 1947 \\
\hline $\begin{array}{l}\text { J. A. Marinsky, L. E. } \\
\text { Glendenin, and C. D. } \\
\text { Coryell }\end{array}$ & $\begin{array}{l}\text { The discovery of promethium, an element found in nature, is attributed to ion } \\
\text { exchange. }\end{array}$ & 1947 \\
\hline $\begin{array}{l}\text { D. K. Hale, D. } \\
\text { Reiechenberg, N. E. } \\
\text { Topp, and C. G. } \\
\text { Thomas }\end{array}$ & Development of carboxylic addition polymers as weak acid cation exchangers. & 1949-1956 \\
\hline $\begin{array}{l}\text { R. M. Barrer and D. } \\
\text { W. Breck }\end{array}$ & New zeolites as molecular sieves with ion exchange properties. & 1951-1956 \\
\hline $\begin{array}{l}\text { H. P. Gregor, K. W. } \\
\text { Pepper, and L. R. } \\
\text { Morris }\end{array}$ & Invention and development of chelating polymers. & 1952-1971 \\
\hline $\begin{array}{l}\text { M. A. Peterson, H. A. } \\
\text { Sober }\end{array}$ & Development of cellulose ion exchangers. & 1956 \\
\hline F. Helfferich & Foundation laid for the new theoretical treatment of ion exchange. & 1959 \\
\hline $\begin{array}{l}\text { T. R. E. Kressmann } \\
\text { and J. R. Millar }\end{array}$ & Invention and development of isoporous ion exchange resins. & 1960 \\
\hline J. Weiss & Thermally regenerable ion-exchange and water desalination based on them. & 1964 \\
\hline
\end{tabular}




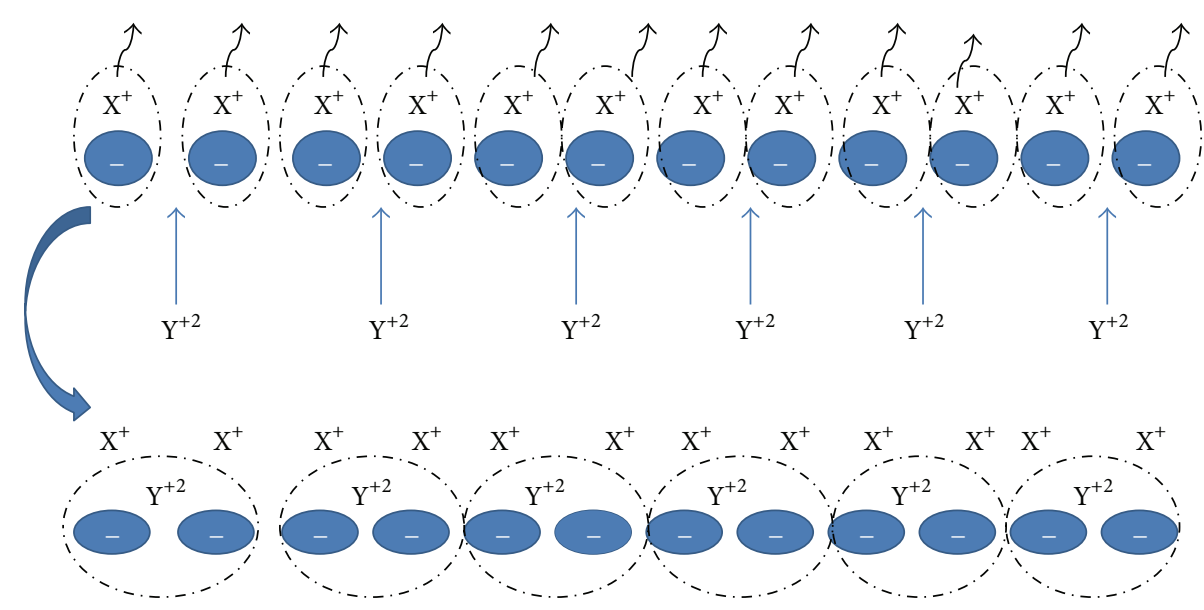

(a)

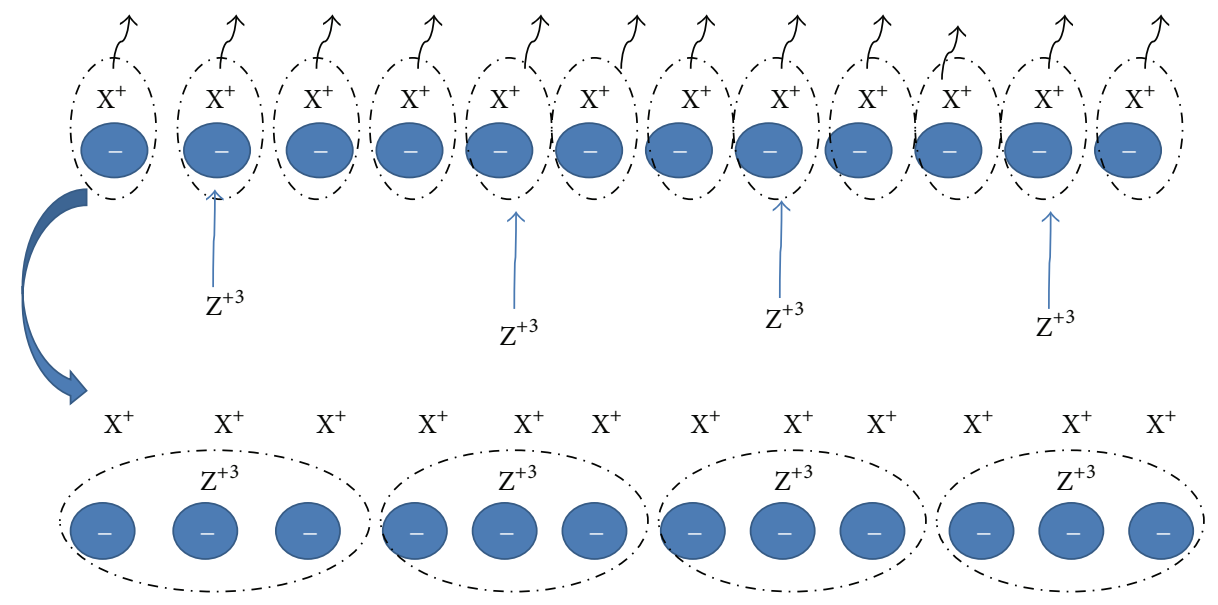

(b)

FIGURE 1: ((a) and (b)) Equivalent character of ion exchange. The following reactions are considered $\overline{\mathrm{RX}}+\mathrm{Y}^{2+}=\overline{\mathrm{RY}}+2 \mathrm{X}^{+}$and $\overline{\mathrm{RX}}+\mathrm{Z}^{3+}=$ $\overline{\mathrm{RZ}}+3 \mathrm{X}^{+}$.

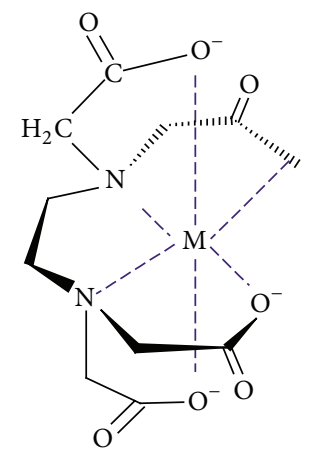

FIGURE 2: Coordination of a metal ion with ethylene diamine tetra acetic acid (EDTA).

1.5. Natural Inorganic Products. Many natural mineral compounds, such as clays (e.g., bentonite, kaolinite, and illite), vermiculite, and zeolites (e.g., analcite, chabazite, sodalite, and clinoptilolite) exhibit ion exchange properties. Natural zeolites were the first materials to be used in ion exchange

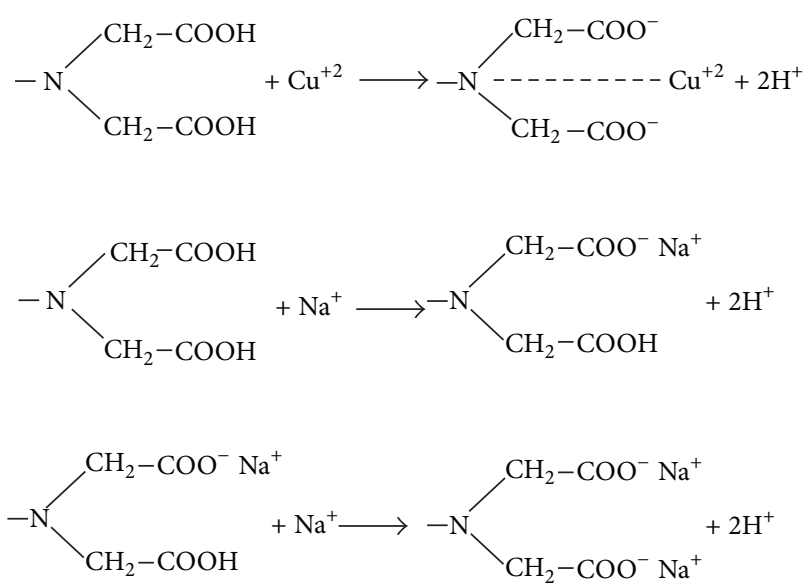

FIgURE 3: Functional groups containing oxygen as electron donor.

processes. Clay materials are often employed as backfill or buffer materials for radioactive waste disposal sites because of their ion exchange properties, low permeability, and easy 


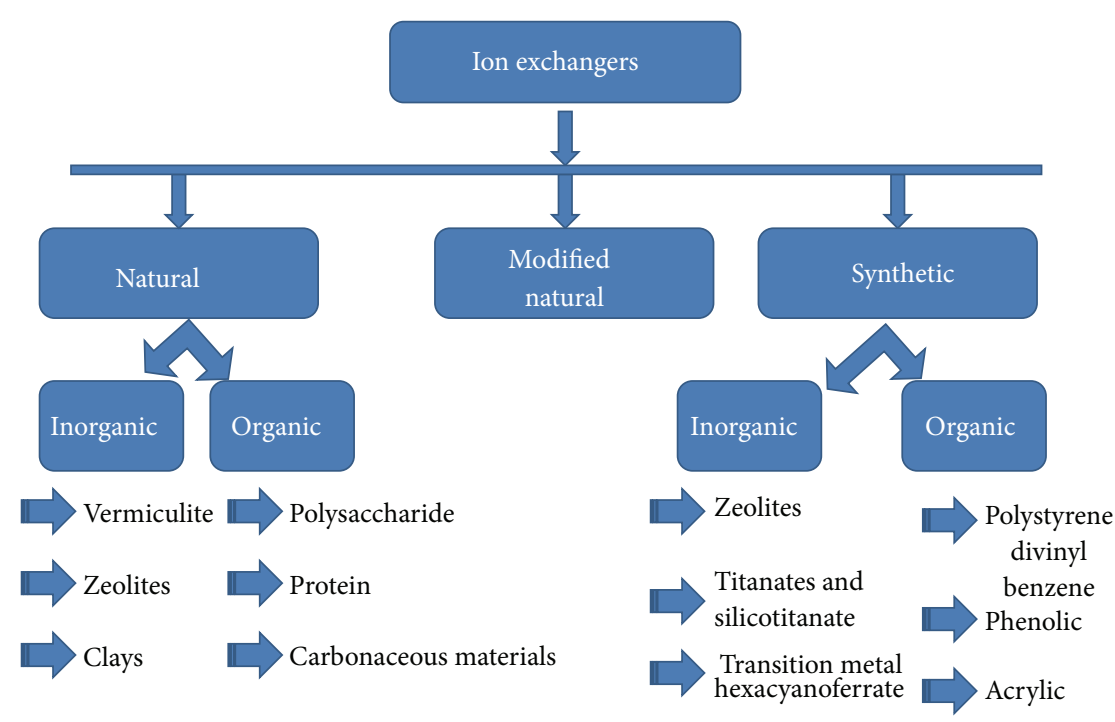

FIgURE 4: Classification of ion exchangers.

workability. Clays can also be used in batch ion exchange processes but are not generally suited to column operation because their physical properties restrict the flow through the bed.

1.6. Modified Natural Ion Exchangers. To improve exchange capacity and selectivity, some naturally occurring organic ion exchangers are modified; for example, cellulose based cation exchangers may be modified by the introduction of phosphate, carbonic, or other acidic functional groups.

The sorption parameters of natural materials can be modified by a chemical and/or thermal treatment; for example, by treating clinoptilolite with a dilute solution of acids or some salts, a more selective form of sorbent can be developed for a particular radionuclide [11].

In Japan natural minerals treated with alkaline solutions under hydrothermal conditions have been proposed for the sorption of caesium and strontium from solution. These treatments have provided materials with distribution coefficients of 1000 to 10000 . Good results have been reported for the removal of caesium and strontium by neoline clays modified with phosphoric acid [12].

1.7. Synthetic Organic Ion Exchangers. In 1935, two chemists at the National Chemical Laboratory in Teddington, Adams and Holmes [7], demonstrated that organic ion exchange resins could be synthesized in a manner similar to the well identified resin "Bakelite," which was prepared by Baekeland in 1909. "Bakelite" is a hard, insoluble condensation resin polymer and can be easily made by heating together phenols and formaldehyde, in the presence of acid or base with the elimination of water. Basically the reaction takes place in two stages (Figure 5).

The repeat of the pervious reactions will generate a threedimensional structure containing weakly acidic phenolic $-\mathrm{OH}$ or OR groups (Figure 6).
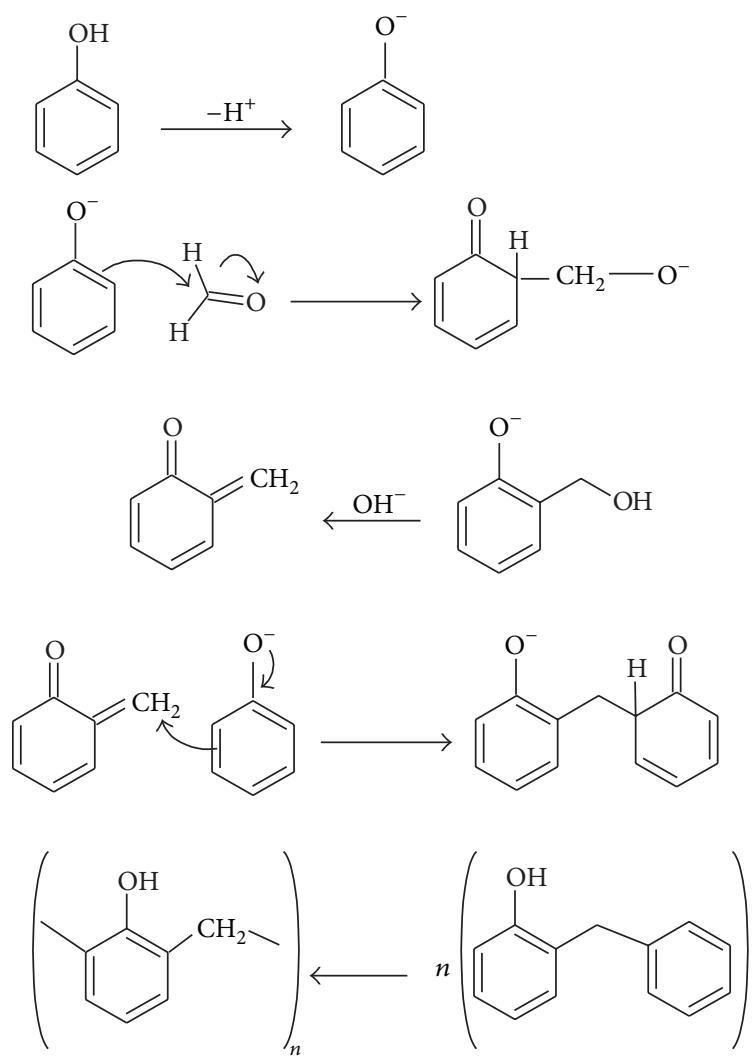

FIGURE 5: Mechanism of formation of Bakelite.

Adams and Holmes showed that these exchanged their hydrogen ions in alkaline solution; they also prepared cation exchanger which worked in acid solution by introducing strongly acidic sulphonic acid groups, $-\mathrm{SO}_{3} \mathrm{H}$, into the structure. This was done either by sulphonation of the final product or by using as starting material not a phenol but a phenolsulphonic acid, in which the sulphonic acid group was already present. 
<smiles>Cc1cc2cc(c1O)Cc1cc(cc(C)c1O)Cc1cc(O)cc(c1O)Cc1cc(O)cc(c1O)Cc1cc(O)cc(c1O)Cc1cc(cc(C)c1O)C2</smiles>

Figure 6: Cross-linked polymer of phenol and formaldehyde.<smiles>C=Cc1ccccc1</smiles>

FIGURE 7: Styrene.

By a suitable modification of their synthesis they were also able to introduce basic groups derived from amines and so prepare synthetic anion exchangers. For the first time it became possible to prepare under controlled conditions both cation and anion exchange resins, the behaviour and stability of which were considerably in advance of other materials.

Until the development of modern techniques of high polymer chemistry, the condensation resins were used successfully. In 1944 d'Alelio, in the United States, produced superior materials. D'Alelio's resins, as well as most of their descendants, were based on a regular three-dimensional network formed by polymerizing the benzene derivative styrene (see Figure 7).

The double bond in the side chain may be opened and styrene units linked end to end to give the polymer chain (Figure 8).

Here, the repeating unit based on the styrene molecule occurs millions of times. Styrene may also be regarded as a derivative of ethylene, in which one hydrogen atom has been replaced by a phenyl group, $-\mathrm{C}_{6} \mathrm{H}_{6}$. Just as ethylene may be polymerized to polyethylene (polythene), styrene readily polymerizes to polystyrene. The chains may be linked together into two- and three-dimensional structures if styrene is mixed with a small proportion of divinyl benzene (Figure 9) which resembles styrene in its properties but has two unsaturated side chains through which it can polymerize.

The polymer formed from a mixture of styrene and divinyl benzene consists of chains like those mentioned previously, linked to another chain wherever the unit is a divinyl benzene molecule instead of styrene (Figure 10).

In the areas enclosed by the broken lines the chains are linked together, and if we imagine this occurring in three dimensions it is easy to see that the resulting polymer will form a fairly regular network (Figure 10). The links between

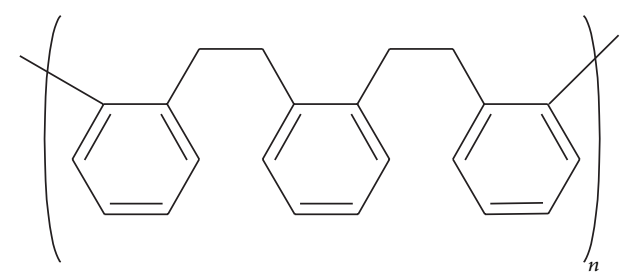

FIGURE 8: Polystyrene.<smiles>C=Cc1ccc(C=C)cc1</smiles>

Figure 9: Divinyl benzene.<smiles>CCCC(CC(C)c1ccc(C(C)CC)cc1)CC(CC(CC(CC)c1ccccc1)c1ccccc1)c1ccc(C(CC(C)c2ccccc2)CC(C)c2ccccc2)cc1</smiles>

FIgURe 10: Polymer of divinyl benzene.

chains are termed "cross-links," and the resin is generally characterized by the percentage of divinylbenzene (DVB) used in its preparation; thus, a 2 percent cross-linked resin contains 2 percent of DVB and 98 percent of styrene.

D'Alelio prepared such resins by emulsion polymerization, using suitable emulsifiers, from which the resin settles as spherical beads. The polymer itself does not work as ion exchanger, but it can be sulphonated just as can the condensation resins, introducing the strongly acidic group, like $\mathrm{SO}_{3} \mathrm{H}$, into the benzene rings of the polymer. The hydrogen ions of this group will exchange with other cations in solution.

Remembering that one benzene ring is repeated many times throughout the polymer, the basic steps in the introductions of the exchanging groups (or "functional" groups as they are usually called) are as shown in Figure cation exchange (a) and anion exchange (b) (Figure 11).

The second of these reactions gives a quaternary ammonium salt, which is strongly ionized, and is readily converted to the corresponding base. The anion readily exchanges with other anions at all $\mathrm{pH}$ values. The cation forms part of an insoluble polymer chain or network, so that the exchanger as a whole is insoluble in water. 


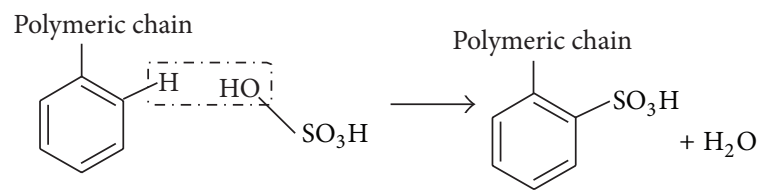

(a)<smiles>O=C(OCc1ccccc1)C(=O)c1ccccc1CCl</smiles>

$+$

$+\left(\mathrm{CH}_{3}\right)_{3} \mathrm{~N}$

FIGURE 11: (a) Cation exchange; (b) anion exchange.

The synthetic ion exchangers like polystyrene-based resins, which form the basis of most resins available commercially today, possess numerous advantages over earlier natural organic exchangers:

(1) the method of synthesis is more controlled, and the end product is more uniform in particle size, degree of cross-linking, and the content of functional groups;

(2) by suitable variation of these factors a wide range of different products may be prepared;

(3) the product is spherical, unlike the irregular particles formed when the solid mass of condensation resin is crushed and sieved. This minimizes waste; in addition, spherical particles pack more uniformly into beds than do irregularly shaped particles. This influences the flow pattern of solutions through the beds and makes it easier to control their behavior;

(4) the physical and chemical stability of these resins are superior to that of the condensation resins.

\subsection{Synthetic Inorganic Ion Exchangers}

1.8.1. Synthetic Zeolites. Zeolites are crystalline, hydrated aluminosilicates of alkali and alkaline earth metals, having infinite, three-dimensional atomic structures [13]. They are further characterized by the ability to lose and gain water reversibly and to exchange certain constituent atoms, also without major change of atomic structure. Along with quartz and feldspar minerals, zeolites are three-dimensional frameworks of silicate $\left(\mathrm{SiO}_{4}\right)$ tetrahedra in which all four corner oxygens of each tetrahedron are shared with adjacent tetrahedra. If each tetrahedron in the framework contains silicon as its central atom, the overall structure is electrically neutral, as is quartz $\left(\mathrm{SiO}_{2}\right)$. In zeolite structures, some of the quadricharged silicon is replaced by triply charged aluminum, giving rise to a deficiency of positive charge. The charge is balanced by the presence of singly-and doubly-charged atoms, such as sodium $\left(\mathrm{Na}^{+}\right)$, potassium $\left(\mathrm{K}^{+}\right)$, ammonium $\left(\mathrm{NH}^{+}\right)$, calcium $\left(\mathrm{Ca}^{2+}\right)$, and magnesium $\left(\mathrm{Mg}^{2+}\right)$, elsewhere in the structure. The empirical formula of a zeolite is of the type

$$
\mathrm{M}_{2 / n} \mathrm{O} \cdot \mathrm{Al}_{2} \mathrm{O}_{3} \cdot x \mathrm{SiO}_{2} \cdot y \mathrm{H}_{2} \mathrm{O},
$$

where $\mathrm{M}$ is any alkali or alkaline earth atom, $n$ is the charge on that atom, $x$ is the ratio of silica and alumina varies number from 2 to 10 , and $y$ is a number from 2 to 7 . The chemical formula for clinoptilolite, a common natural zeolite is

$$
\left(\mathrm{Na}_{3} \mathrm{~K}_{3}\right)\left(\mathrm{Al}_{6} \mathrm{Si}_{40}\right) \mathrm{O}_{96} \cdot 24 \mathrm{H}_{2} \mathrm{O} \text {. }
$$

Atoms or cations (i.e., charged metal atoms) within the second set of parentheses are known as structural atoms, because with oxygen they make up the rigid framework of the structure. Those within the first set of parentheses are known as exchangeable ions, because they can be replaced (exchanged) more or less easily with other cations in aqueous solution, without affecting the aluminosilicate framework. This phenomenon is known as ion exchange or more commonly cation exchange. The exchange process involves replacing one singly-charged exchangeable atom in the zeolite by one singly-charged atom from the solution or replacing two singly-charged exchangeable atoms in the zeolite by one doubly-charged atom from the solution. The magnitude of such cation exchange in a given zeolite is known as its cation exchange capacity (CEC) and is commonly measured in terms of moles of exchangeable cation per gram (or 100 grams) of zeolite or in terms of equivalents of exchangeable cations per gram (or 100 grams) of zeolite.

1.8.2. Polybasic Acid Salts. These are the acidic salts of multivalent, mainly quadrivalent, metals formed by mixing acidic oxides of metals belonging to IV, V, and VI groups of periodic table. They have nonstoichiometric composition based on condition of precipitation. Specially tetravalent metal acid (tma) salts $[14,15]$ are gaining importance due to their excellent thermal stability and chemical resistivity. They are cation exchangers, possessing the general formula $\mathrm{M}(\mathrm{IV})\left(\mathrm{HXO}_{4}\right) 2$ $\times n \mathrm{H}_{2} \mathrm{O}$, where $\mathrm{M}(\mathrm{IV})=\mathrm{Zr}$, Ti, Sn, Ce, Th, and so forth and $\mathrm{X}=\mathrm{P}, \mathrm{W}, \mathrm{As}, \mathrm{Mo}, \mathrm{Sb}$, and so forth. These materials possess structural hydroxyl groups, the $\mathrm{H}$ of the $-\mathrm{OH}$ being the exchangeable sites. They possess an appreciable ion exchange capacity as well as high selectivity for certain metal ions. Tma salts can be obtained in both amorphous and crystalline forms. Though structures of crystalline materials are well known, they have not been found to be suitable in column operations as they are obtained in powder forms. Amorphous materials on the other hand can be obtained in granular form, very suitable for column operations [16]. Tma salts being synthesized by sol-gel routes, materials with varying water content, composition, ion exchange capacity, and crystallinity can be obtained by varying parameters such as stoichiometry and concentration of the reagents used, temperature at which they are mixed, rate of addition, mode of mixing, and $\mathrm{pH}$. Ion exchange materials with higher selectivities are continuously being investigated [17].

1.8.3. Hydrous Oxides. The hydrous oxides of some metal ions are also used as ion exchange materials like freshly precipitated trivalent metal oxides. 
1.8.4. Metal Ferrocyanides. Insoluble metal ferrocyanides are also used as inorganic ion exchange materials commonly known as scavengers for alkali metals. They are easy to prepare and are used widely in radioactive waste treatment.

1.8.5. Insoluble Ion Exchange Materials. They are of great interest as ion exchangers and are being prepared largely by precipitation from metal salt solution with sodium sulphide and hydrogen sulphide.

1.8.6. Heteropolyacids. HPAs are complex proton acids that incorporate polyoxometalate anions (heteropolyanions) having metal-oxygen octahedra as the basic structural units [10]. The heteropoly compounds are quite strong oxidizing agent. Heteropoly acids are used as ion exchangers derived from 12 HPAs with general formula $\mathrm{H}_{n} \mathrm{XY}_{12} \mathrm{O}_{40} \cdot n \mathrm{H}_{2} \mathrm{O}$. The first characterized and the best known of these is the Keggin heteropolyanion typically represented by the formula $\mathrm{XM}_{12} \mathrm{O}_{40}^{x-8}$ where $\mathrm{X}$ is the central atom $\left(\mathrm{Si}^{4+}, \mathrm{P}^{5+}\right.$, etc. $), x$ is its oxidation state, and $\mathrm{M}$ is the metal ion $\left(\mathrm{Mo}^{6+}\right.$ or $\left.\mathrm{W}^{6+}\right)$.

\section{Mechanism of Ion Exchange Process}

A most common ion exchange system includes a water swollen ion exchange material and surrounding aqueous solution. Ion exchange like any heterogeneous process is accomplished by transfer of ions to and from the interphase boundary; that is, the chemical reaction itself, diffusion inside the material, and diffusion in the surrounding solution should be considered as ion exchange. Beside the two major phases, the thin film of solution at surface of the exchanger should be considered separately. The film properties differ from properties of the surrounding bulk solution. Formation of thin film is unavoidable. Even rigorous agitations only reduce the thickness of the interphase film.

Figure 12 shows the simplest illustration of the mass transfer at ion exchange. Ions B diffuse from the solution through the film into beads, and ions A diffuse out of the beads crossing the film into the solution. This interphase diffusion of counterions is called ion exchange. Counterions are exchangeable ions carried by ion exchangers. Counterions can freely move within the frame work, but their movement has to be compensated by corresponding counter movements of other ions of the same change fulfilling the electroneutrality principle. The term counterion has two interpretations.

(a) It can be used exclusively for ions inside the ion exchanger.

(b) It can be used in a broad sense whether in the exchanger or in the external solution; all ionic species with charge sign opposite to that of the functional groups can be called counterions.

Coion is a term used for ionic species with the same charge sign as the functional group. Ionic form is the term defining which counterion is present in the ion exchange.

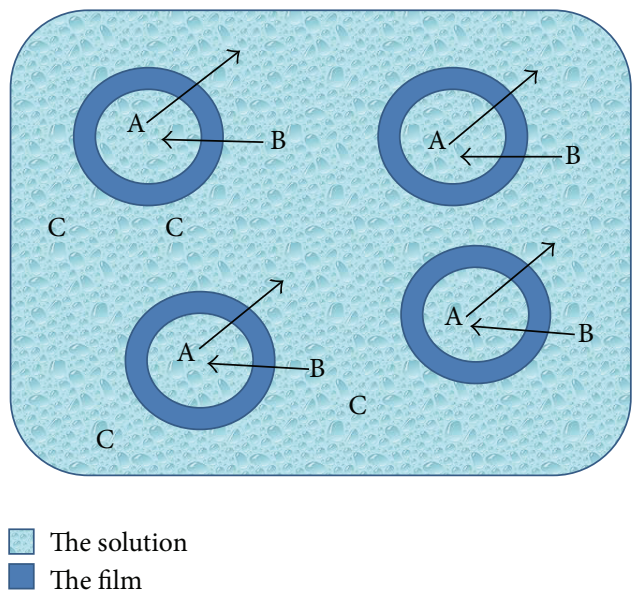

Figure 12: Mass transfer during ion exchange: the material is initially bonded with counterion A. Solution initially contains counterion B and coion C. During the process, ions B are transferred inside the exchanger replacing ions $\mathrm{A}$. When transferred between two phases the ions pass through the film which cannot be removed with agitation.

Any counterion which leaves the ion exchanger is replaced by an equivalent amount of other counterions. This is a consequence of the electroneutrality requirement. When a counterion crosses the interphase boundary, an electric potential is created between two phases. This potential must be compensated by the movement of another counterion in opposite direction (ion exchange) or by the movement of co-ion in the same direction. In many systems, particularly in diluted solution, Donnan exclusion (Donnan exclusion: reduction in concentration of mobile ions within an ion exchange membrane due to the presence of fixed ions of the same sign as the mobile ions) restricts entering of coions into the exchanger during the whole process. In this case, coions do not participate in the reaction and have little effect on the rate. However, an indirect influence can occur.

From Figure 13 it is clear that initially the dissolved salt dissociates containing the first ion step (1), followed by diffusion of the first ion from solution towards the interphase film step (2). Then the ion diffuses out of the film step (3) towards the material phase step (4). The ion associates with the functional group step (5) and then dissociation of second ion and the functional group occurs in step (6). The second ion diffuses inside the material phase towards the surface step (7). The second ion diffuses inside the interphase film step (8), followed by diffusion and random distribution in the solution step (9). Thus the formation of second ion pair completes step (10).

2.1. Selectivity of Ion Exchange Materials. Selectivity is a parameter that is defined at specific solution conditions. Other equilibrium associated with the ion of interest may affect the selectivity of the ion. Three general classes of models have been invoked for explaining exchange selectivity.

Nonmechanistic models were derived using principles of mass action and thermodynamics, mechanistic models based on diffuse double layer theory, and mechanistic models 


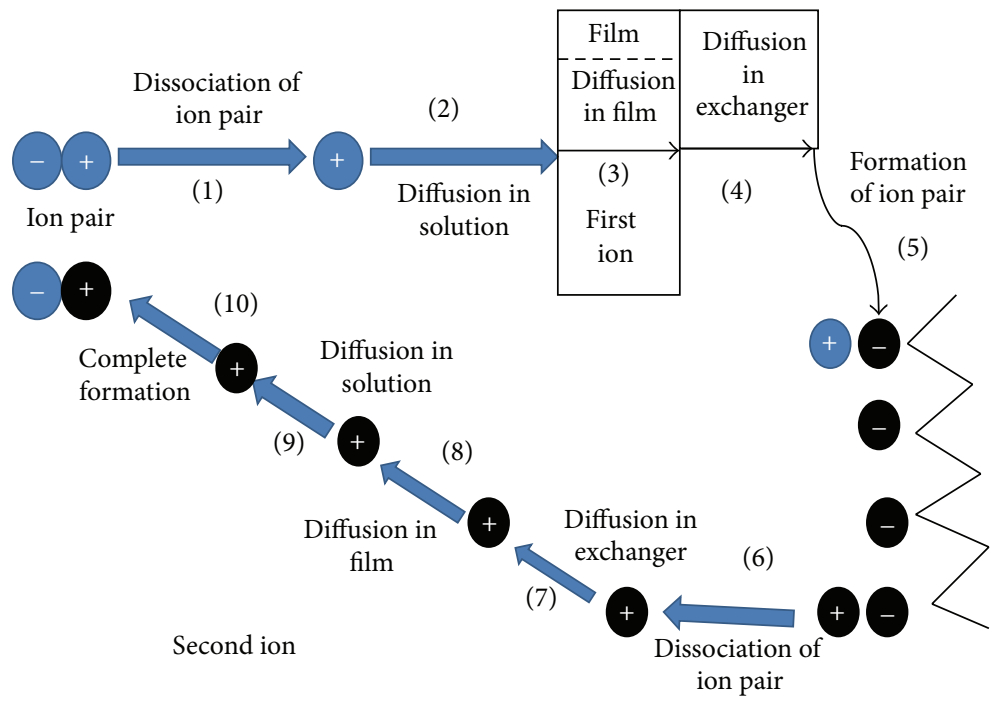

FIGURE 13: Schematic representation of various steps involved in ion exchange process [10].

derived from the energetics of hydration and electrostatic interactions.

Calculation of mass action selectivity coefficients from measured ion exchange data is perhaps the most common treatment of cation exchange selectivity. Ideally, such coefficients estimate thermodynamic equilibrium constants $(K \sim)$. This treatment of ion exchange does not distinguish between the solid and liquid phases and therefore requires that activities be determined for adsorbed ions. Vanselow (1932) suggested that activities of adsorbed ions are closely approximated by mole fractions of the ions adsorbed onto the solid [18]. Measured Vanselow coefficients $(K v)$, however, often vary with the ionic composition of the exchanger and the total ionic strength of the equilibration solution [19]. Gaines and Thomas (1953) proposed integration of measured selectivity coefficients over the full compositional range as a means of estimating thermodynamic equilibrium constants [20]. Under the Gaines-Thomas approach, variations in measured selectivity coefficients with the ionic composition of the exchanger are arbitrarily attributed to variations in activities of the adsorbed ions. The Gaines-Thomas approach explicitly includes changes in chemical potential for water that may occur during an ion exchange reaction.

The thermodynamic treatment of cation exchange selectivity has 2 fundamental problems: (1) hysteresis is sometimes observed in ion exchange reactions involving $2: 1$ phyllosilicates [21], and when hysteresis is observed the thermodynamic treatment of ion exchange is invalid, because reversibility is a requirement of equilibrium. (2) The standard thermodynamic approach is inherently nonmechanistic: one may predict the outcome of an ion exchange reaction, but one will never know the cause of ion exchange selectivity.

Eriksson proposed a mechanistic model for cation exchange selectivity that is based on diffuse double layer (DDL) theory [22]. With various modifications, the Eriksson model has been shown to estimate selectivity coefficients for heterovalent exchange reactions that are within an order of magnitude of measured coefficients [19, 23]. The Eriksson model considers only electrostatic interactions and treats all cations as point charges; therefore, it does not distinguish selectivities for exchange reactions involving cations of like charge. Several authors have proposed modifications of the Eriksson model in an effort to overcome this problem. Neal and Cooper, for example, assumed that an ion could come no closer to a charged surface than its hydrated radius [24], and Nir used specific binding coefficients to account for differences in selectivity for cations of like charge [25]. For a given clay, the Eriksson model can be adjusted to fit measured data. However, no amount of adjustment will change the fundamental prediction of the Eriksson model that higher charged cations will be increasingly preferred as layer charge of the clay increases. Although this relationship has been qualitatively demonstrated for $\mathrm{Na}-\mathrm{Ca}$ exchange [23], the opposite relationship is observed for $\mathrm{K}-\mathrm{Ca}$ exchange $[26,27]$. Failure of the Eriksson model to account for the relationship between layer charge and selectivity in $\mathrm{K}-\mathrm{Ca}$ exchange reactions suggests a major flaw in the Eriksson model.

On a more fundamental level, the validity of the Eriksson model for describing the ion exchange selectivity exhibited by smectites may be questioned because the Eriksson model is based on DDL theory. In aqueous systems, smectites exist as quasicrystals. Each quasicrystal consists of 1 to several hundred lamellae stacked with parallel $c$ axes but randomly oriented $a$ and $b$ axes. Diffuse double layers may form in the bulk solution adjacent to external surfaces of the quasicrystals; however, DDLs do not form between lamellae within a quasicrystal. Within a quasicrystal, the lamellae are separated by 4 or fewer discrete layers of water molecules, and anions are excluded from the interlayer spaces [28]. Assuming that an average quasicrystal consists of 10 coordinated lamellae with layer charge distributed uniformly over all surfaces, then 
$90 \%$ of the exchangeable cations are retained on internal surfaces and only $10 \%$ are retained on external surfaces. Diffuse double layer theory and the Eriksson model are relevant for describing selectivity exhibited by the $10 \%$ of cations sorbed on the external surfaces, but have no bearing on selectivity for the $90 \%$ of cations retained on internal surfaces.

Another approach to understanding cation exchange selectivity is based on Eisenman's theory, which was developed to explain the behavior of ion selective electrodes [29]. Eisenman argued that the molar free energy of exchange (AGex) is determined by the net change in Coulombic and hydration energies for the exchanging cations. Eberl applied Eisenman's theory to 2:1 phyllosilicates for exchange reactions between Cs and other alkali cations [30]. To do so, he assumed that Cs was unhydrated both in solution and when adsorbed onto a clay. However, he was unable to use the Eisenman approach for partially hydrated 2:1 clays, because the hydration states of partially hydrated interlayer cations were unknown. Instead, he assumed that the interlayer water in a $2: 1$ clay was analogous to water in a concentrated alkali halide solution. With this assumption, he was able to estimate $A G \sim$ as a function of both interlayer molarity and layer charge. Consistent with experimental data, the analysis indicated that preference for the lesser hydrated cation increases as interlayer water decreases and as layer charge density increases.

\section{Kinetics of ion Exchange Materials}

Kinetics is dependent on these considerations.

(i) Mass transfer (diffusion) in the solution or in other external medium is an unavoidable step in any ion exchange interaction. The process is well known. It can be easily assisted by hydrodynamic turbulences and thus cannot be considered as a limiting step for ion exchange.

(ii) The film is a solution zone of certain thickness with no convection. The mass transfer in the film is defined solely by the diffusion coefficients. The film thickness can be reduced by an agitation.

(iii) Mass transfer in the exchange phase depends on the physiochemical properties of the system and cannot be enhanced without altering the chemical system itself.

(iv) Reaction between counterions and fixed groups are the only chemical interaction that can affect the overall rate of the ion exchange.

(v) Complexes dissociation and formation of ion pairs in solution is not considered as a part of the ion exchange.

As followed from these considerations most of the ion exchange processes are purely diffusion phenomenon; that is, they are controlled by diffusion of the counterions rather than by actual chemical reaction.

Two main rate determining steps are considered in most of the cases: diffusion of ions inside the material or diffusion of ions through the liquid film. In both cases migration of ions is defined solely by properties of the system. Rate of these steps cannot be enhanced or slowed down with external action without altering the system itself.

Diffusion inside the material is referred as particle diffusion, and diffusion through film is referred as film diffusion. In simple cases the rate of ion exchange is determined by the slower of these two processes. Dominance of one of these mechanisms can be predicted using approximate [31-33]. For particle diffusion control, see [32-34]

$$
\begin{aligned}
& \frac{Q \cdot \bar{D} \cdot \delta}{C \cdot D \cdot r_{0}} \cdot\left(5+2 \alpha_{B}^{A}\right) \ll 1, \\
& \frac{Q \cdot \bar{D} \cdot \delta}{C \cdot D \cdot r_{0}} \cdot\left(5+2 \alpha_{B}^{A}\right) \gg 1 .
\end{aligned}
$$

And for film diffusion control, see [34], where $Q$ is the concentration of fixed group and $C$ is solution concentration. $\bar{D}$ is the interdiffusion coefficient in the ion exchanger, $D$ is the interdiffusion coefficient in the film, $r_{0}$ is the bead radius, $\delta$ is the film thickness, and $\alpha_{B}^{A}$ is the separate factor.

According to criterion all factors, which tend to increase the rate of interdiffusion in the beads and to reduce the rate in the film, favor the film diffusion control and vice versa. Thus, the film diffusion control may prevail in system characterized by the following properties [31]:

(i) high concentration of exchange sites,

(ii) low degree of cross-linking,

(iii) small particle size,

(iv) dilute solution,

(v) inefficient agitation.

The simplest technique for distinguishing between particle and film diffusion control is interruption test. The ion exchange interaction is performed in batch mode. The conversion degree is monitored. The beads are removed from the solution much before the completion of the process. After a brief period the material is reimmersed in the same solution. If the concentration gradient is created inside the beads, these gradients level out when supply of fresh ions from the solution is interrupted. If the process is controlled by the particle diffusion, the rate immediately after the reimmersion is greater prior to the interruption. In case of the film diffusion, the amount of the ions unevenly distributed in the film at the moment of the interruption is almost undetectable, and no concentration gradient in the bead exists. Thus, the interruption does not affect this process and the rate before and after the interruption in the same. 


\section{Mathematical Approach to Kinetics of Ion Exchange}

The general approach to metal ion exchange kinetics is to apply conventional diffusion equations. Due to accounting of interaction for ion exchange makes modeling highly complicated. These interactions are diffusion induced electric forces selectivity specific interactions, changes in swelling. The basis for description of diffusion processes is Fick's first law [35]:

$$
J_{i}=-D_{i} \operatorname{grad} C_{i}
$$

(see [35]). For phase of ion exchange

$$
\bar{J}_{i}=-\bar{D}_{i} \operatorname{grad} \bar{C}_{i}
$$

(see [35]), where $J_{i}$ is the flux of the ion in moles/time and unit cross-section, $D$ is the diffusion coefficient, and $C_{i}$ is the concentration in moles per unit volume.

In the simplest case, the diffusion coefficient is taken as constant, and thus, the flux is proportional to the concentration gradient. $D$ being constant means that the species is not subject to any forces beside the concentration inhomogeneity. This is not common case when ion exchange is considered because of electrocoupling of ionic fluxes. Influence of coupling can be neglected only when fluxes are equal. There can be exception in ion exchange. An example of such exception is diffusion controlled isotope in systems where concentration of others are leveled. The corresponding diffusion coefficient (same for both isotopes) is referred as the self-diffusion coefficient. Its value is constant throughout any single phase of the system but of course depends on the nature and composition of the particular phase and on the temperature.

In most of the other cases, the diffusion coefficient is not constant. It means that the variation of the diffusion coefficient must be taken into account. The influence of electric field can be accounted by using the Nernst-Planck equation, instead of Fick's equation:

$$
J_{I}=-D_{i} \operatorname{grad} C_{i}-D_{i} Z_{i} C_{i}\left(\frac{F}{R T}\right) \operatorname{grad} \varepsilon
$$

(see [35]), where $\varepsilon$ is electric potential.

If two exchanged ions are present in the phase with different concentration, their diffusion is affected by the electrocoupling interaction unequally. As shown in (7), the flux of diffusion species depends only on the concentration gradient and does not depend on the concentration itself (first term in (7)), because the diffusion is purely statistical phenomenon involving no physical force on the molecular level.

In contrast, the electric field causes a true physical force acting on every ion and producing a flux proportional to the concentration of the respective ion (second term in (8)). As a result, the electric field mainly affects the ions present as in majority. For those in the minority, the second term is small and thus (8) approaches (6). As a result, the ions present in small concentration migrate essentially at the rate of their ordinary diffusion. For the interdiffusion of two counterions, in the absence of a significant co-ion transfer, the two fluxes are rigorously coupled. Diffusion of the majority ion is not much affected by the action of electric field and thus controls the rate.

Equation (6) is not only possible way to describe diffusion process. The theory of irreversible thermodynamics states that the driving force for diffusion is not the concentration gradient but the gradient chemical potential.

In this case

$$
J_{i}=-D_{i}^{\prime} \frac{C_{i}}{R T} \operatorname{grad} \mu_{i}
$$

(see [35]), where $\mu_{i}$ is the chemical potential and $D_{i}^{\prime}$ is interconnected with $D$ by equation:

$$
D_{i}=-D_{i}^{\prime}\left(1+\frac{\delta \ln \gamma_{i}}{\delta \ln C_{i}}\right)
$$

(see [35]), while $D_{i}$ depends on the concentration and thus can differ in different points of the nonhomogenous medium. $D_{i}^{\prime}$ can be assumed to be having a constant value. If the system contains more than two compounds (9) should be replaced with

$$
J_{i}=-\sum_{J=1}^{n} D_{J \cdot i}^{\prime} \frac{C_{i}}{R T} \operatorname{grad} \mu_{j},
$$

(see [35]), where $n$ is the number of compounds. However due to the electrocoupling of ionic fluxes only one diffusion flux can be considered as independent, and thus (9) could be used for most of the ion exchange [10].

In conclusion, the concentration of the external solution can be decisive for the rate determining step; that is, a simple increase of solution concentration could alter the mechanism from film diffusion to particle diffusion control.

\section{Conclusion}

Ion exchange is an ancient technique documented more than hundred years ago. Since then they were used for softening water to an incomparable wider scale of applications and has become an integral part of new technical and industrial processes. There is a wide diversity of ion exchange materials. They have many appearances, natural and synthetic, organic and inorganic, cationic, anionic and amphoteric, and so forth. A most common ion exchange system includes a water swollen ion exchange materials and surrounding systems. In all cases the mechanism involves formation of thin film. During the interphase diffusion the electroneutrality is maintained. The kinetics of ion exchange involves mass transfer which is solely defined by the diffusion coefficients. This depends upon physicochemical properties of system. The two main steps considered to be influencing the rate are diffusion of ions inside the material or diffusion of ions through liquid film. The most simple technique for distinguishing the two is interruption test. 
The paper suggests about steps targeting to enhance the rates of the ion exchange process. The most straightforward way to get faster diffusion inside the exchanger beads is to select materials with low density of the gel. Overall, the rate of process can be enhanced even without replacement of the materials. Reducing the bead size can reduce the time of the equilibrium achievement for particle controlled process. Elevated temperature enhances the rates independent of the rate controlling step.

\section{References}

[1] J. Pérez-Arantegui, J. Molera, A. Larrea et al., "Luster pottery from the thirteenth century to the sixteenth century: a nanostructured thin metallic film," Journal of the American Ceramic Society, vol. 84, no. 2, pp. 442-446, 2001.

[2] S. Padovani, C. Sada, P. Mazzoldi et al., "Copper in glazes of renaissance luster pottery: nanoparticles, ions, and local environment," Journal of Applied Physics, vol. 93, no. 12, pp. 1005810063, 2003.

[3] S. Padovani, I. Borgia, B. Brunetti et al., "Silver and copper nanoclusters in the lustre decoration of Italian Renaissance pottery: an EXAFS study," Applied Physics A, vol. 79, no. 2, pp. 229$233,2004$.

[4] H. S. Thompson, “On the absorbent power of soils," Journal of the Royal Agricultural Society of England, vol. 11, pp. 68-74, 1850.

[5] J. T. Way, "On the power of soils to absorb manure," Journal of the Royal Agricultural Society, vol. 11, pp. 313-379, 1850.

[6] R. Gans, "Zeolites and similar compounds, their constitution and meaning for technology and agriculture," Jahrbuch der Königlich Preussischen Geologischen Landesanstalt, vol. 26, p. 179, 1905.

[7] B. A. Adams and E. L. Holmes, "Adsorptive properties of synthetic resins," Journal of Chemical Society, vol. 54, pp. 1-6, 1935.

[8] http://dardel.info/IX/other_info/history.html.

[9] C. A. Lucy, "Evolution of ion-exchange: from moses to the manhattan project to modern times," Journal of Chromatography A, vol. 1000, no. 1-2, pp. 711-724, 2003.

[10] A. A. Zagorodni, Ion Exchange Materials Properties and Applications, Elseiver, Oxford, UK, 1st edition, 2007.

[11] N. B. Chernjatskaja, "Sorption of strontium on clinoptilolite and heulandite," Radiochemistry, vol. 27, pp. 618-621, 1988.

[12] N. Yamasaki, S. Kanahara, and K. Yanagisawa, "Adsorptions of strontium and cesium ions on hydrothermally altered minerals of feldspar," Lithia-Mica and Bauxite, Nippon Kagaku Kaishi, vol. 12, pp. 2015-2018, 1984.

[13] M. Naushad, "Inorganic and composite ion exchange materials and their applications," Ion Exchange Letters, vol. 2, pp. 1-14, 2009.

[14] K. G. Varshney and A. M. Khan, "Amorphous inorganic ion exchangers," in Inorganic Ion Exchangers in Chemical Analysis, M. Qureshi and K. G. Varshney, Eds., pp. 177-270, CRC Press, 1991.

[15] A. Clearfield, "Inorganic ion exchangers: a technology ripe for development," Industrial and Engineering Chemistry Research, vol. 34, no. 8, pp. 2865-2872, 1995.

[16] G. Alberti, U. Costantino, and M. L. Luciani, "Crystalline insoluble acid salt of tetravalent metals. II.Comparison of ionexchange properties of crystalline $\alpha$-zirconium phosphate and $\alpha$-titanium phosphate," Gazzetta Chimica Italiana, vol. 110, p. 61, 1980.

[17] T. Möller, Selective crystalline inorganic materials as ion exchangers in the treatment of nuclear waste solutions [Academic Dissertation], University of Helsinki, Helsinki, Finland, 2002.

[18] A. P. Vanselow, "Equilibria of the base-exchange reactions of bentonites, permutites, soil colloids, and zeolites," Soil Science, vol. 33, no. 2, pp. 95-113, 1932.

[19] H. Laudelout, R. van Bladel, G. H. Bolt, and A. L. Page, "Thermodynamics of heterovalent cation exchange reactions in a montmorillonite clay," Transactions of the Faraday Society, vol. 64, pp. 1477-1488, 1968.

[20] G. L. Gaines Jr. and H. C. Thomas, "Adsorption studies on clay minerals. II. A formulation of the thermodynamics of exchange adsorption," The Journal of Chemical Physics, vol. 21, no. 4, pp. 714-718, 1953.

[21] K. Verburg and P. Baveye, "Hysteresis in the binary exchange of cations on 2:1 clay minerals: a critical review," Clays \& Clay Minerals, vol. 42, no. 2, pp. 207-220, 1994.

[22] E. Eriksson, "Cation exchange equilibria on clay minerals," Soil Science, vol. 74, no. 2, pp. 103-113, 1952.

[23] A. Maes and A. Cremers, "Charge density effects in ion exchange: I. Heterovalent exchange equilibria," Journal of the Chemical Society, Faraday Transactions, vol. 73, pp. 1807-1814, 1977.

[24] C. Neal and D. M. Cooper, "Extended version of Gouy-Chapman electrostatic theory as applied to the exchange behavior of clay in natural waters," Clays \& Clays Minerals, vol. 31, no. 5, pp. 367-376, 1983.

[25] S. Nir, "Specific and nonspecific cation adsorption to clays: solution concentrations and surface potentials," Soil Science Society of America Journal, vol. 50, no. 1, pp. 52-57, 1986.

[26] B. L. Sawhney, "Cesium uptake by layer silicates: effect on interlayer collapse and cation exchange capacity," in Proceedings of the International Clay Conference, L. Heller, Ed., pp. 605-611, Israel University Press, Tokyo, Japan, September 1969.

[27] I. Shainberg, N. I. Alperovitch, and R. Keren, "Charge density and Na-K-Ca exchange on smectites," Clays \& Clay Minerals, vol. 35, no. 1, pp. 68-73, 1987.

[28] A. M. Posner and J. P. Quirk, "The adsorption of water from concentrated electrolyte solutions by montmorillonite and illite," Proceedings of the Royal Society A, vol. 278, no. 1372, pp. 35-56, 1964.

[29] G. Eisenman, "Cation selective glass electrodes and their mode of operation," Biophysical Journal, vol. 2, no. 2, pp. 259-323, 1962.

[30] D. D. Eberl, "Alkali cation selectivity and fixation by clay minerals," Clays \& Clay Minerals, vol. 28, no. 3, pp. 161-172, 1980.

[31] F. Helfferich, Ion Exchange, McGraw Hill, New York, NY, USA, 1962.

[32] F. G. Helfferich and Y. L. Hwang, "Ion Exchange kinetics," in Ion Exchangers, K. Dorfner, Ed., pp. 1277-1309, Walter de Gruyter, Berlin, Germany, 1991.

[33] F. G. Helfferich, "Ion exchange kineticsevolution of a theory," in Mass Transfer and Kinetics of Ion Exchange, L. Liberti and F. G. Helfferich, Eds., pp. 157-180, Martinus Nijhoff Publishers, Hague, The Netherlands, 1983.

[34] F. Helfferich, "Kinetik des Ionenaustauschs," Angewandte Chemie, vol. 68, no. 22, pp. 693-698, 1956. 
[35] G. V. Samsonov, E. B. Trostyanskaya, and G. E. El'kin, Ion Exchange, Sorption of Organic Substances. (Ionnyi Obmen, Sorbtsiya Organicheskikh Veshchestv), Nauka, Leningrad, Russia, 1969. 

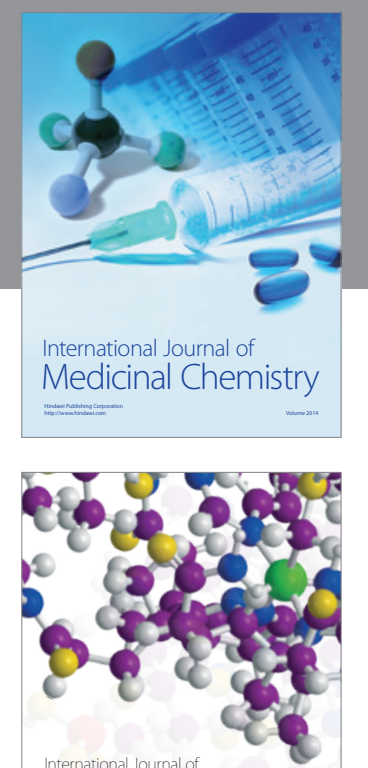

\section{Carbohydrate} Chemistry

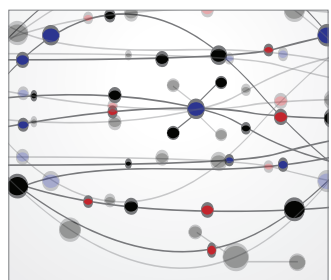

The Scientific World Journal
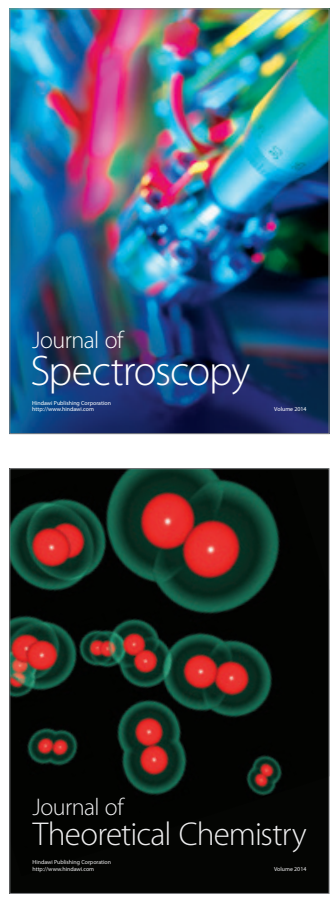
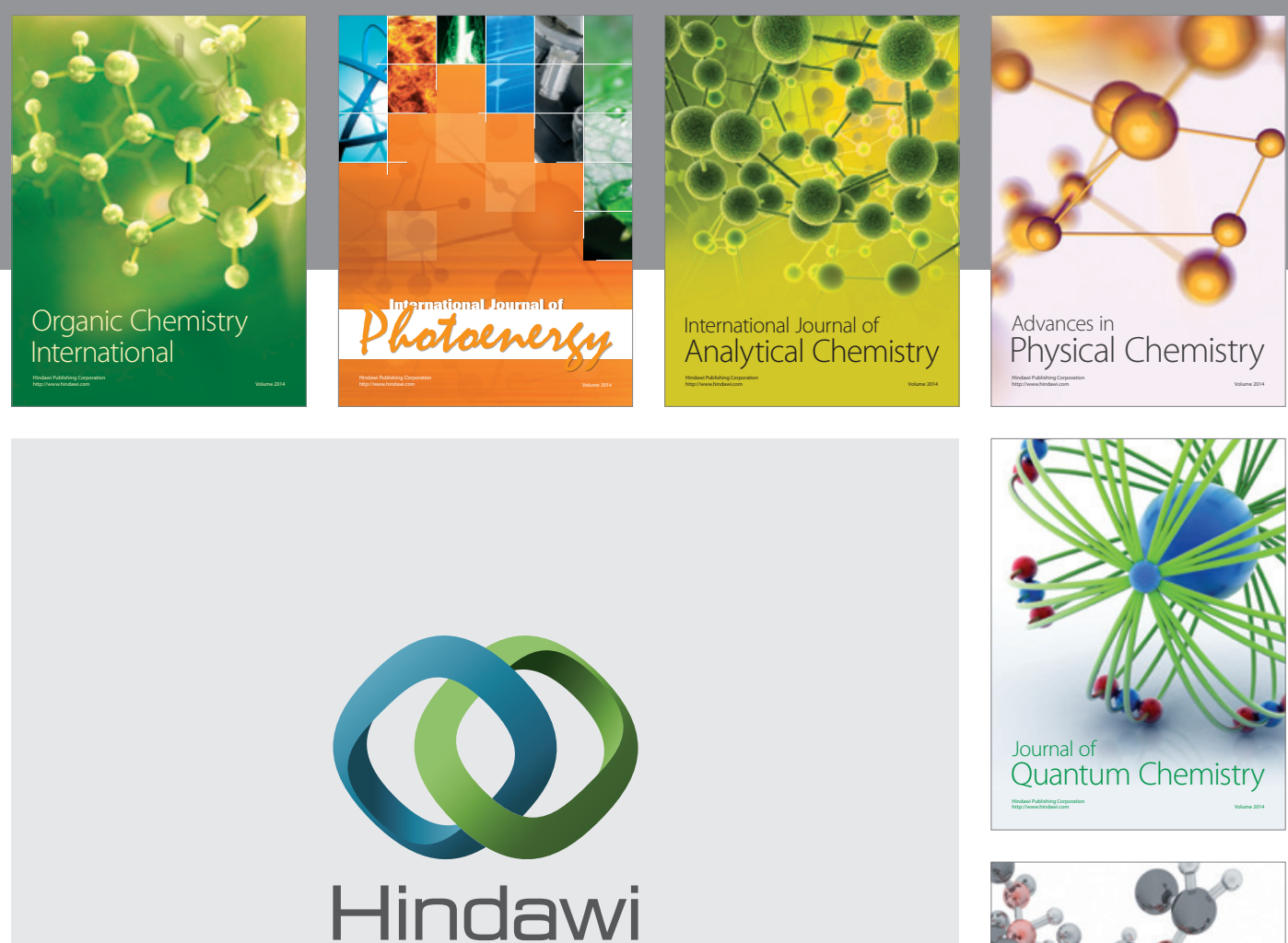

Submit your manuscripts at

http://www.hindawi.com

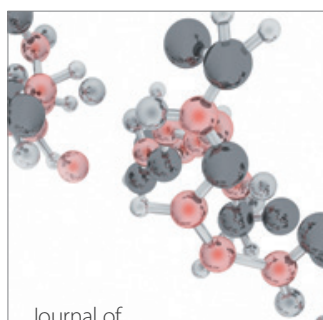

Analytical Methods

in Chemistry

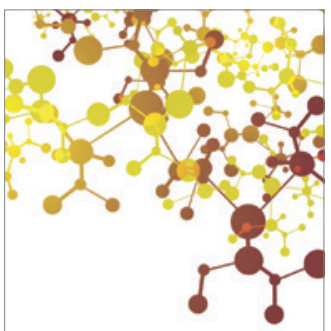

Journal of

Applied Chemistry

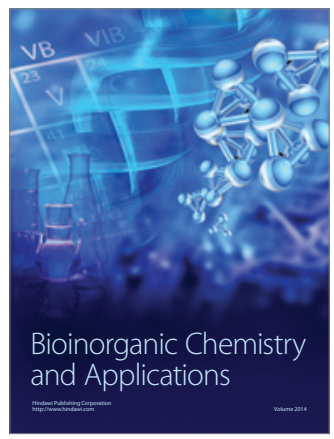

Inorganic Chemistry
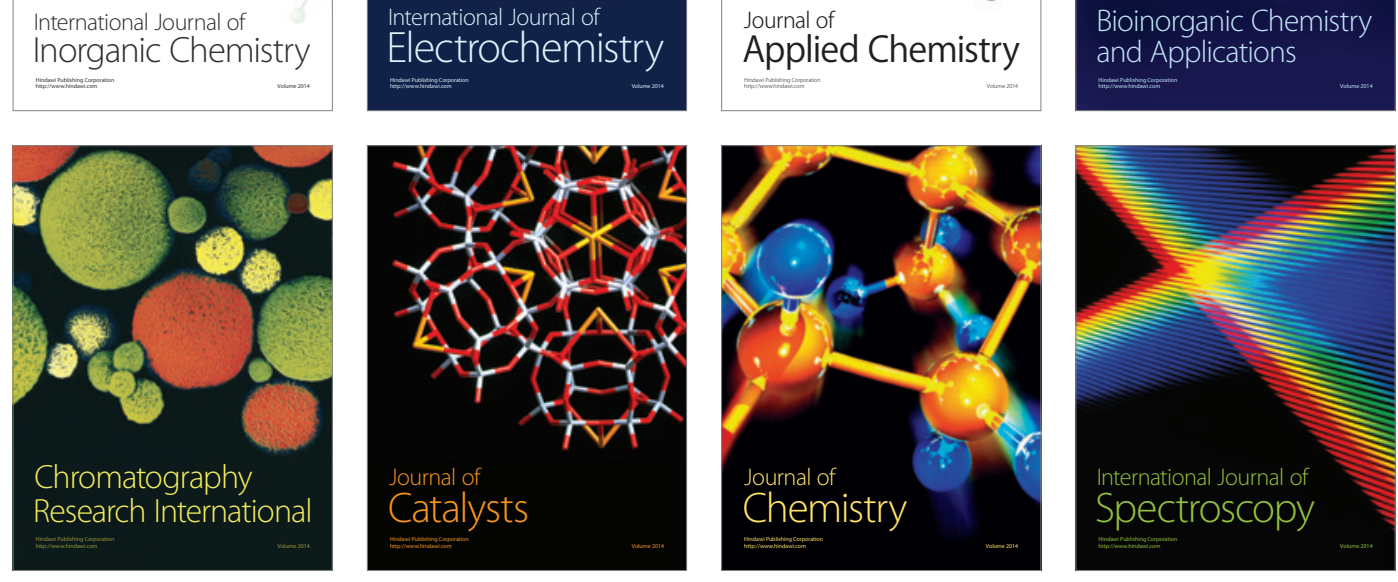